\title{
Instrumento digital para medição de diâmetro florestal usando microcontrolador de
}

\section{baixo custo}

\author{
Digital instrument for diameter forest measurement using low-cost microcontroller \\ Instrumento digital para la medición del diámetro de los bosques mediante microcontrolador de \\ bajo costo
}

Recebido: 01/12/2021 | Revisado: 06/12/2021 | Aceito: 13/12/2021 | Publicado: 21/12/2021

\author{
Marina Sbardella \\ ORCID: https://orcid.org/0000-0002-5052-5847 \\ Universidade Estadual Paulista, Brasil \\ e-mail: marina.sba@hotmail.com \\ José Rafael Franco \\ ORCID: https://orcid.org/0000-0002-7129-4304 \\ Universidade Estadual Paulista, Brasil \\ e-mail: jose.rafael@unesp.br \\ Jonath Weríssimo da Silva Gomes \\ ORCID: https://orcid.org/0000-0002-2669-3541 \\ Universidade Estadual Paulista, Brasil \\ e-mail: jonathwerissimo@gmail.com \\ Alexandre Dal Pai \\ ORCID: https://orcid.org/0000-0002-1283-901X \\ Universidade Estadual Paulista, Brasil \\ e-mail: dal.pai@unesp.br \\ Enzo Dal Pai \\ ORCID: https://orcid.org/0000-0002-0570-8644 \\ Universidade Estadual Paulista, Brasil \\ e-mail: enzo-dal.pai@unesp.br
}

\begin{abstract}
Resumo
Este estudo descreve o processo de criação e montagem de um sistema para aquisição de dados de diâmetro à altura do peito de forma automatizada. A construção do protótipo móvel contou com os seguintes componentes: Arduino Pro mine, Módulo Cartão Micro SD, Sensor de Distância, Display LCD e Módulo Joystick. A montagem do sistema foi realizada em recipiente de plástico transparente. A validação dos dados foi feita por meio da comparação dos valores de diâmetro à altura do peito coletados com a suta convencional e o protótipo construído, por meio de medidas frontais e laterais de um plantio de Eucalyptus grandis. Os resultados demonstraram correlação linear positiva entre os dados obtidos pelo método convencional, utilizando suta mecânica e os dados obtidos atrás do protótipo confeccionado. O coeficiente de correlação de Pearson foi de 0,92 e 0,91 para as medidas frontal e lateral, respectivamente. O projeto se mostra viável para o uso dentro do setor florestal, principalmente em razão do baixo custo para confecção. No entanto, o protótipo apresentado necessita de maiores ajustes, principalmente em sua estrutura, a fim de facilitar o manuseio.
\end{abstract}

Palavras-chave: Arduíno; Inventário florestal; Suta digital; Mensuração florestal.

\begin{abstract}
This study tests the process of creating and assembling a system for automated data acquisition of diameter at breast height. The construction of the mobile prototype included the following components: Arduino Pro mine, Micro SD Card Module, Distance Sensor, LCD Display and Joystick Module. The system was assembled in a transparent plastic pool. Data validation was performed by comparing the diameter at breast height values collected with the conventional bra and the built prototype, through frontal and lateral measurements of a Eucalyptus grandis plantation. The results showed a positive correlation between the data obtained by the conventional method, using a mechanical brace, and the data obtained from the prototype made. Pearson's correlation coefficient was 0.92 and 0.91 for frontal and lateral measurements, respectively. The project proves to be viable for use within the forestry sector, mainly due to the low cost of production. However, the presented prototype needs further adjustments, especially in its structure, in order to facilitate handling.
\end{abstract}

Keywords: Arduino; Forest inventory; Digital caliper; Forest measurement. 


\begin{abstract}
Resumen
Este estudio prueba el proceso de creación y montaje de un sistema para la adquisición automatizada de datos de diámetro a la altura del pecho. La construcción del prototipo móvil incluyó los siguientes componentes: mini Arduino Pro, módulo de tarjeta Micro SD, sensor de distancia, pantalla LCD y módulo de joystick. El sistema se montó en una piscina de plástico transparente. La validación de los datos se realizó comparando los valores de diámetro a la altura del pecho recolectados con el sostén convencional y el prototipo construido, mediante mediciones frontales y laterales de una plantación de Eucalyptus grandis. Los resultados mostraron una correlación positiva entre los datos obtenidos por el método convencional, utilizando una abrazadera mecánica, y los datos obtenidos del prototipo realizado. El coeficiente de correlación de Pearson fue de 0,92 y 0,91 para las medidas frontal y lateral, respectivamente. El proyecto demuestra ser viable para su uso dentro del sector forestal, principalmente debido al bajo costo de producción. Sin embargo, el prototipo presentado necesita más ajustes, especialmente en su estructura, para facilitar su manejo.
\end{abstract}

Palabras clave: Arduino; Inventario forestal; Sujetador digital; Medición forestal.

\title{
1. Introdução
}

Uma das principais etapas que envolvem o manejo florestal é o inventário florestal. Os inventários florestais fornecem os subsídios necessários para o planejamento das atividades de exploração e do manejo e são divididos em: inventário de reconhecimento ou diagnóstico, destinados a análise da composição e a estrutura da floresta, inventário a $100 \%$, ou préexploratório, onde o intuito de predizer o estoque de madeira existente nos compartimentos de manejo a partir do levantamento de $100 \%$ da área de interesse e o inventário contínuo, ou de monitoramento, cujo objetivo é e acompanhar o desenvolvimento estrutural de uma floresta ao longo do tempo por meio de mensurações sucessivas (Araujo, 2006).

Em inventários florestais, uma das variáveis dendrométricas utilizadas é o diâmetro a altura do peito (DAP). O DAP é uma variável de obtenção fácil e direta, e a partir dela, é possível realizar a estimativa de diversas outras grandezas de relevância no setor florestal, como a área transversal, área basal e o volume madeira contidos na floresta (Freitas \& Wichert, 1998; Sanquetta et al., 2009). Sua medida é obtida por meio de instrumentos como fitas métricas e diamétricas, sutas mecânicas e eletrônicas, que são alocadas a 1,30m de altura em relação ao nível do solo (Corte et al., 2016).

A escolha do equipamento a ser utilizado irá depender da praticidade, custo e a finalidade da medição. Apesar de a fita métrica ser muito utilizada em inventários tanto de espécies florestais nativas como em plantios comerciais, o uso da suta tornou-se comum, por minimizar os déficits de convexidade e isoperimétrico a partir da medição do diâmetro de dois eixos do fuste a uma determinada altura (Gonçalves et al., 2009), o que torna a medida mais precisa em comparação aos demais. É válido ressaltar que, o erro de um centímetro na determinação do diâmetro pode afetar em até $19 \%$ no cálculo do volume da floresta (Couto et al., 1989). Segundo Cunha et al. (2002), os erros mais frequentes que ocorrem durante a medição do DAP, estão relacionados ao mau uso dos equipamentos de medição pelo operador.

Com o passar das décadas e com o aumento no número de hectares de florestal plantadas, a fim de minimizar e ao mesmo tempo acelerar o trabalho de coleta de dados em campo, reduzir equipes de trabalhos, além aumentar a precisão na coleta dos dados, foram desenvolvidas sutas eletrônicas. Esses equipamentos avançados contam com diversos acessórios exclusivos, como sensores de distância por ultrassom, GPS, teclado, laser, Bluetooth, entre outras funcionalidades que permitem a automatização da coleta dos dados.

Embora o uso de equipamentos eletrônicos em atividades de mensuração florestal facilite tanto a coleta como a manipulação dos dados (Freitas \& Wichert, 1998), o uso de sutas eletrônicas é pouco presenciado quando se trata de pequenos e médios produtores de madeira, uma vez que estes equipamentos apresentam elevado custo de aquisição e estão comumente relacionados a softwares e protocolos proprietários, tornando-se pouco flexíveis (Ribeiro et al., 2016).

Em contrapartida, o uso de microcontroladores Arduíno está se tornando cada vez mais comum, por constituir de uma plataforma baseada em hardware e software livre para as áreas de automação e robótica, na qual é possível adicionar diversos componentes e programá-los para diferentes atividades (Silva et al., 2014). Conforme o guia elaborado pela 
Multilógica Shop (2016), as montagens rápidas com ajuda de uma protoboard e componentes básicos de eletrônica são uteis em diferentes setores, pois auxiliam na elaboração de protótipos muitas vezes de baixo custo e de fácil confecção.

Com base no exposto, o objetivo do presente estudo foi construir um protótipo de suta digital de baixo custo, utilizando microcontroladores Arduino e sensores para captação e armazenamento de diâmetro de DAP de espécies florestais.

\section{Metodologia}

A construção deste protótipo seguiu a metodologia de pesquisa quantitativa, conforme Pereira et al., 2018. O protótipo foi planejado a partir de pesquisas feitas sobre componentes e sensores necessários para o seu bom funcionamento, em seguida construído e utilizado na coleta de dados de DAP e comparado com dados obtidos por método de mensuração florestal convencional.

\subsection{Componentes utilizados}

Para a montagem do protótipo experimental móvel foram utilizados os seguintes componentes: Arduino Pro mine, Módulo Cartão Micro SD, Sensor de Distância, Display LCD e Módulo Joystick.

O Arduino Pro mine (Figura 1a) é um hardware de código aberto para construir aplicações. Trata-se de uma versão econômica do modelo UNO, possuindo os mesmos componentes, sendo mais barato e menor, utilizando o microcontrolador ATmega328, opera em 3V ou 5V com 14 entradas e saídas digitais e 8 analógicas, 32KB de memória Flash, 2KB SRAM, 1KB EEPROM, velocidade do clock de $8 \mathrm{MHz}$ (operante a $3 \mathrm{~V}$ ) $16 \mathrm{MHz}$ (operante a $5 \mathrm{~V}$ ). Esta placa foi desenvolvida para pequenos projetos permitindo a ligação de vários componentes (Arduino, 2020; Campos, 2015).

O módulo Cartão Micro SD (Figura 1e) permite a conexão de um micro cartão SD ao Arduino por meio da interface SPI (protocolo de comunicação usado para leitura e gravação de dados na memória flash do cartão), o modulo é compacto, eficiente e fácil de utilizar para sistemas de datalogger e armazenamento de dados (Straub, 2018; Vidal, 2017).

O sensor de distância (Figura 1c) modelo VL53L0X mede a distância por meio de laser infravermelho de 30 a 2000mm, controlado através da interface I2C. Este módulo usa a tecnologia FlightSense da ST para medir com precisão a distância por meio do tempo que o pulso de luz leva até colidir com um objeto e ser refletido de volta ao detector (Waveshare, 2018, Nascimento; Silva \& Kashiwagi, 2019).

O Display LCD (Figura 1b) modelo 16x2 Backlight Verde permite uma interface visual para exibir informações do Arduino pela interface I2C exibindo textos, números e símbolos (Murta, 2018).

O módulo Joystick (Figura 1d) modelo KY-023 possui a finalidade de conectar dispositivos eletrônicos com uma espécie de controle de vídeo game, permitindo enviar comandos nos eixos analógicos X e Y (Oliveira, 2020).

O Arduino IDE é um software de código aberto, que permite ao usuário escrever programas e efetuar upload para a placa Arduino via comunicação serial. O código informa ao Arduino o que deve executar durante o funcionamento, a IDE possui a própria linguagem de programação baseada na linguagem C++ (Souza, 2013; Arduino, 2021).

A simulação da montagem dos protótipos foi feita pelo programa de modelagem online Tinkercard, da Autodesk. 
Figura 1. Circuito eletrônico e componente: a) Arduino Pro mine; b) Figura 1b; c) sensor de distância; d) módulo Joystick; e) módulo Cartão Micro SD.

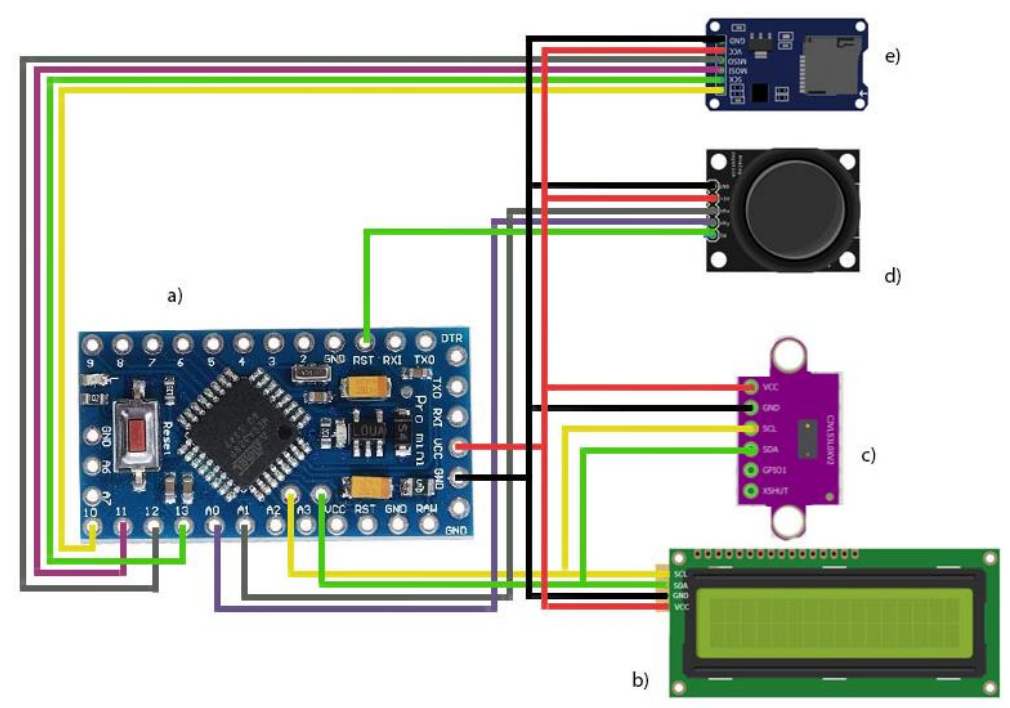

Fonte: Autores.

\subsection{Montagem do protótipo}

A Figura 2 mostra a instalação e ligação dos componentes, visor LCD (Figura 2a), bateria (Figura 2b), módulo cartão micro SD (Figura 2c), joystick (Figura 2d) e recipiente plástico (Figura 2e). Nesta imagem não foi possível registrar o microcontrolador Arduino, pois ele está localizado abaixo dos fios e da bateria.

A estrutura (microcontrolador + sensores) foram conectadas a uma bateria universal portátil USB, da marca Power Bank, 5V de 800aAh. A montagem do sistema foi realizada em recipiente de plástico, modelo retangular, com travas laterais, transparente.

Figura 2. Vista interna do protótipo e localização de seus componentes. 2a) Visor LCD; 2b) Bateria; 2c) módulo cartão micro SD; 2d) joystick; 2e) recipiente plástico.

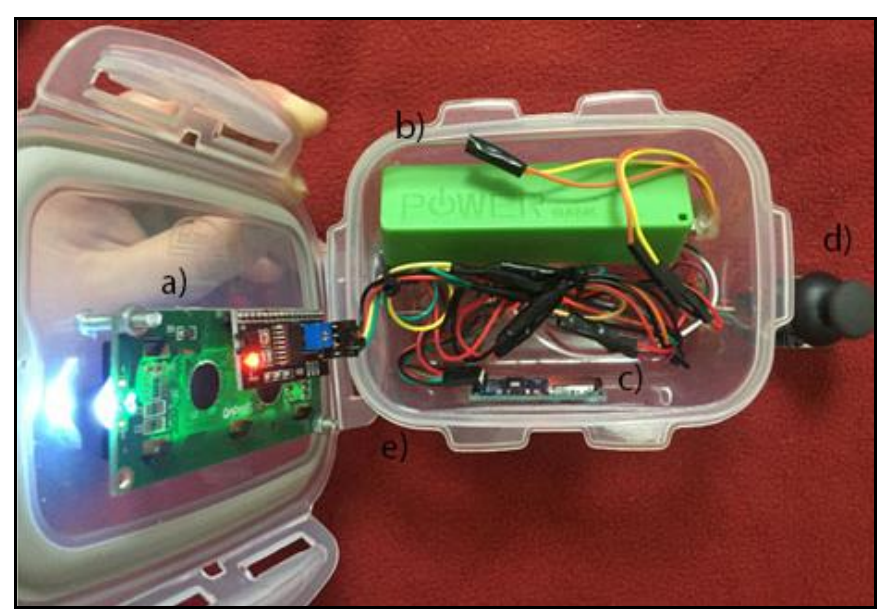

Fonte: Autores.

A Figura 3 exibe o posicionamento do sensor de distância no protótipo. O alvo utilizado para capturar a distância do sensor foi uma estrutura confeccionada com madeira (Figura 3a), posicionada na extremidade do braço fixo da suta, para que o laser emitido pelo sensor batesse na estrutura e calculassem a distância entre objeto é o sensor. 
Figura 3. Vistas superior e lateral do protótipo. a) Vista superior e indicação da localização do sensor; b) Vista lateral.

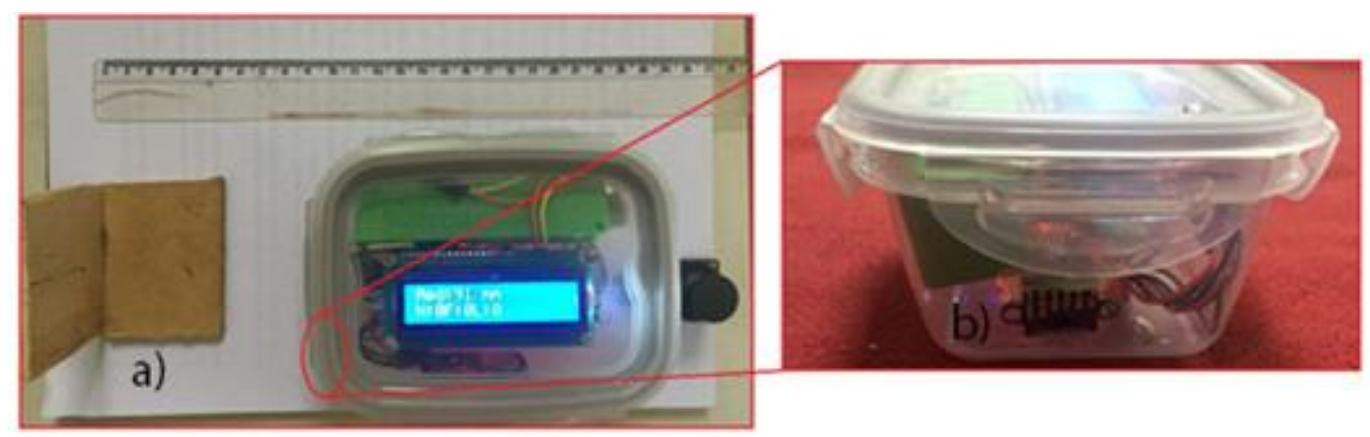

Fonte: Autores.

\subsection{Funcionamentos do sistema}

O fluxograma (Figura 4) exibe o funcionamento do sistema desenvolvido para esta aplicação. Quando o sistema é iniciado, é atribuída à variável cont_arvore o valor 0, representando o valor inicial da contagem de árvores.

As medidas de DAP são divididas em frontal e lateral. A aquisição da medida frontal pode ser feita pressionando o botão joystick para cima, onde o valor pode ser visualizado através do visor LCD em tempo real. A distância entre o sensor e o alvo é dado em mm e pode ser salvo no cartão SD. Os dados são salvos no formato " 0,0 , frontal, 56 ”, onde " 0,0 : representa a contagem da árvore"; "Frontal: representa o tipo da medida" e "56: representa a distância em mm".

A obtenção da medida lateral é feita ao pressionar o botão joystick para a esquerda. Deste modo, os dados podem ser visualizados no visor LCD e são salvos em cartão SD no formato “0,0, Lateral, 4”.

A transição entre a medida de uma árvore e outra pode ser feita pressionando o botão joystick para baixo. Assim, é somado à variável cont_arvore o valor 1 . Sendo assim, o indivíduo 1 é representado pelo conjunto 0,0, indivíduo 2: 1,0, indivíduo 3: 2,0 e assim por diante.

Em caso de erros na aquisição dos dados, tanto frontal como lateral, o usuário pode eliminar o valor. Para isso, basta pressionar o botão joystick para direita e o valor 1 será subtraído da variável cont_arvore.

Caso a árvore apresente mais um fuste ou bifurcação do tronco em até 1,30m do solo as medidas de DAP podem ser salvas na contagem na mesma árvore, como por exemplo: "1,1, frontal, 22”, medida frontal do segundo tronco, "1,1, lateral, 77”, medida lateral do segundo tronco. Após ser realizada a medida de todos os troncos o usuário pressiona o botão do joystick para baixo e realiza a medida da próxima árvore. Pressionando o botão do joystick no centro a contagem de árvores é reiniciada, sem apagar os dados já registrados. 
Figura 4. Fluxograma do sistema.

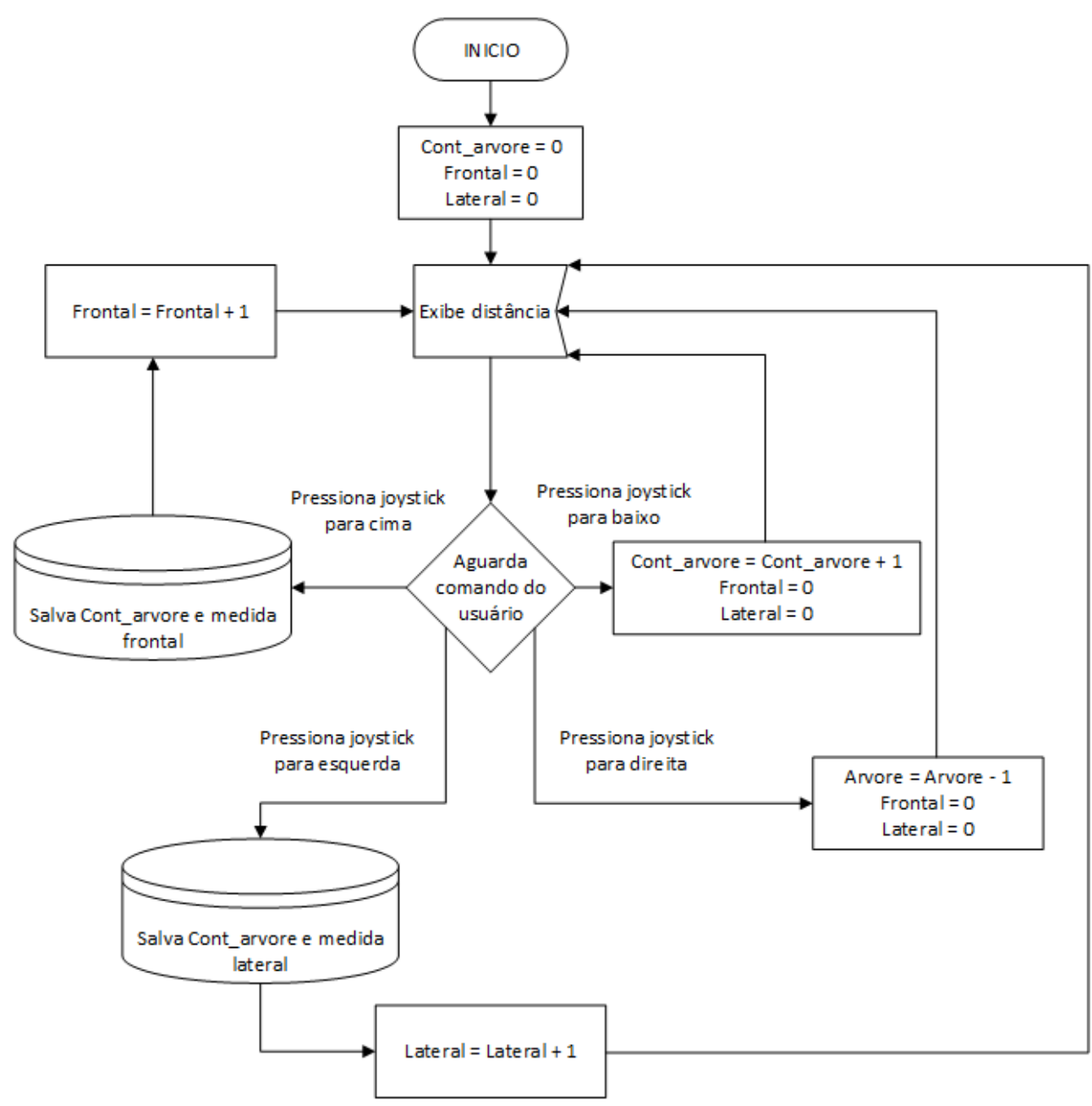

Fonte: Autores.

\subsection{Armazenamento dos dados}

Os dados de DAP coletados foram registrados e armazenados em um módulo MicroSD. Após a coleta das medidas é possível importar os dados em um computador e exibir as informações em qualquer software de planilha eletrônica. Os dados (Figura 5) são armazenados no bloco de notas, obedecendo a uma sequência numérica, como por exemplo, 0,0; 1,0; 2,0..., sendo que o primeiro valor de cada par de dados refere-se ao primeiro diâmetro, chamado de medida frontal e o segundo valor, ao segundo diâmetro, chamado de medida lateral. Caso a árvore apresente mais um fuste ou bifurcação do tronco em até $1,30 \mathrm{~m}$ do solo os dado são gravados 0,$1 ; 0.2, \ldots$ 
Figura 5. Formato de registro e armazenamento de dados em cartão SD e importação em bloco de notas.

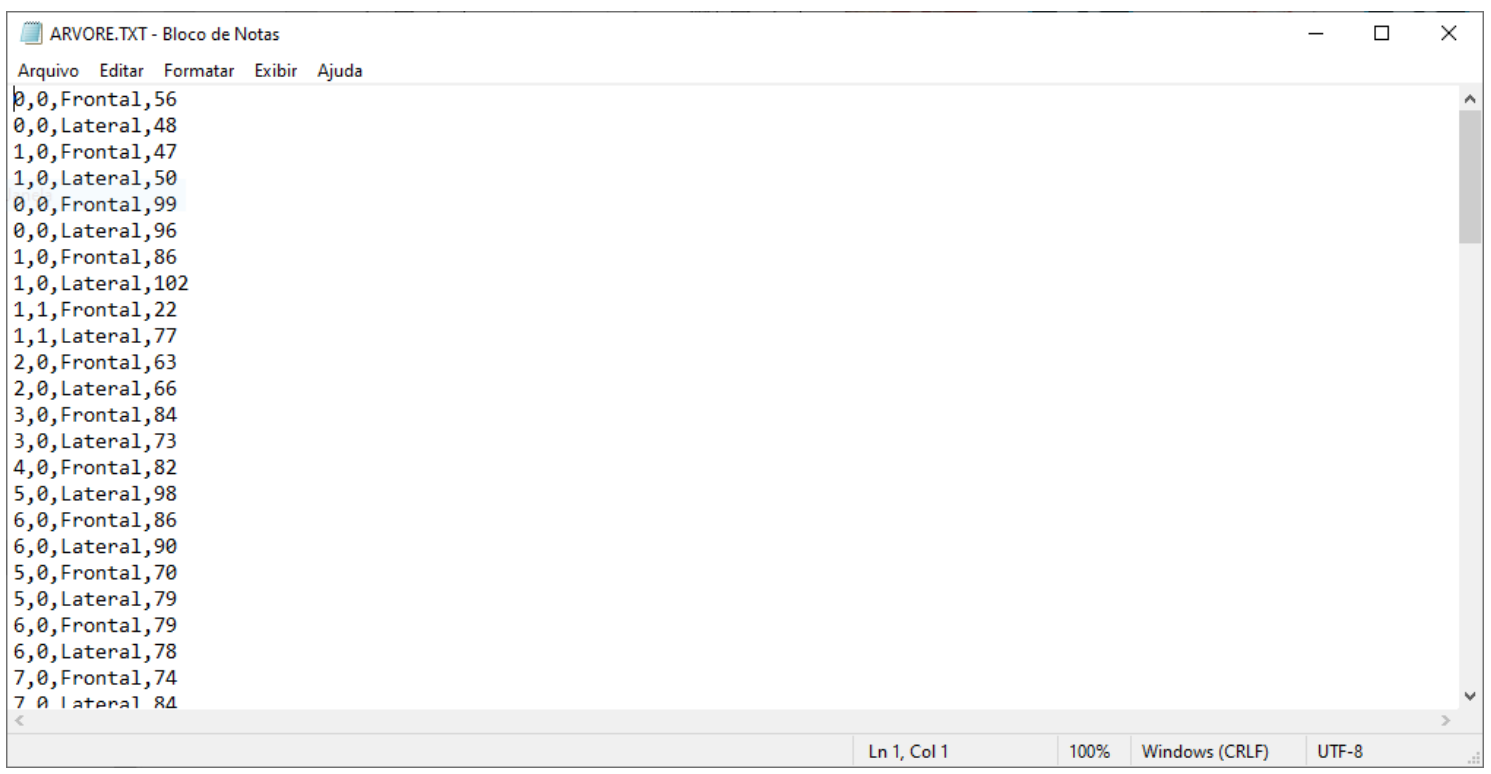

Fonte: Autores.

\subsection{Custos para a criação do protótipo}

O custo estimado para montagem do protótipo, no ano de 2019, como pode ser visto na Tabela 1, foi de $\mathrm{R} \$ 161,38$. Por se tratar de um protótipo móvel, o custo final do instrumento de mensuração florestal envolve gastos para aquisição de uma suta mecânica.

Tabela 1. Detalhamento de componentes, site de aquisição e valor para confecção do protótipo.

\begin{tabular}{llr}
\hline Componente & Site de aquisição & Valor (R\$) \\
\hline Arduino Pro Mine & Felipeflop & 26,90 \\
Módulo Cartão Micro SD & Felipeflop & 9,90 \\
Cartão Micro SD 2GB & Mercado Livre & 18,99 \\
Sensor de Distância & Mercado Livre & 41,89 \\
Display LCD & Felipeflop & 35,90 \\
Joystick & Eletrogate & 7,90 \\
Bateria Universal portátil USB & Eletrogate & 19,90 \\
\hline Total $(\mathbf{R} \$)$ & & 161,38 \\
\hline
\end{tabular}

Fonte: Autores.

\subsection{Validação dos dados}

Após montagem da estrutura (microcontrolador + sensores), o protótipo foi alocado sobre o braço fixo de uma suta mecânica de 650mm (Figura 6). No braço móvel da suta, foi acrescentada uma estrutura de madeira, para servir como barreira para o sensor de distância.

A validação dos dados foi feita por meio da comparação dos valores de DAP coletados com a suta convencional e o protótipo construído. Foram coletados 39 valores de DAP para cada posição (frontal e lateral), para cada um dos instrumentos.

As medidas foram realizadas em um plantio de Eucalyptus grandis manejados em sistema de alto fuste, com aproximadamente 2 anos, localizado em Itatinga (22 $58^{\prime}$ S e ' ${ }^{\circ} 48^{\circ} 43^{\prime} \mathrm{O}$ ), estado de São Paulo. A coleta dos dados foi realizada 
por um único operador. Os dados coletados com protótipo foram armazenados em cartão SD e em seguida baixados no formato txt. A relação entre os dados de DAP obtidos pela suta mecânica e o protótipo construído foi obtido pelo método de correlação de Pearson (r), utilizando o programa R.

Figura 6. Montagem do protótipo em suta convencional.

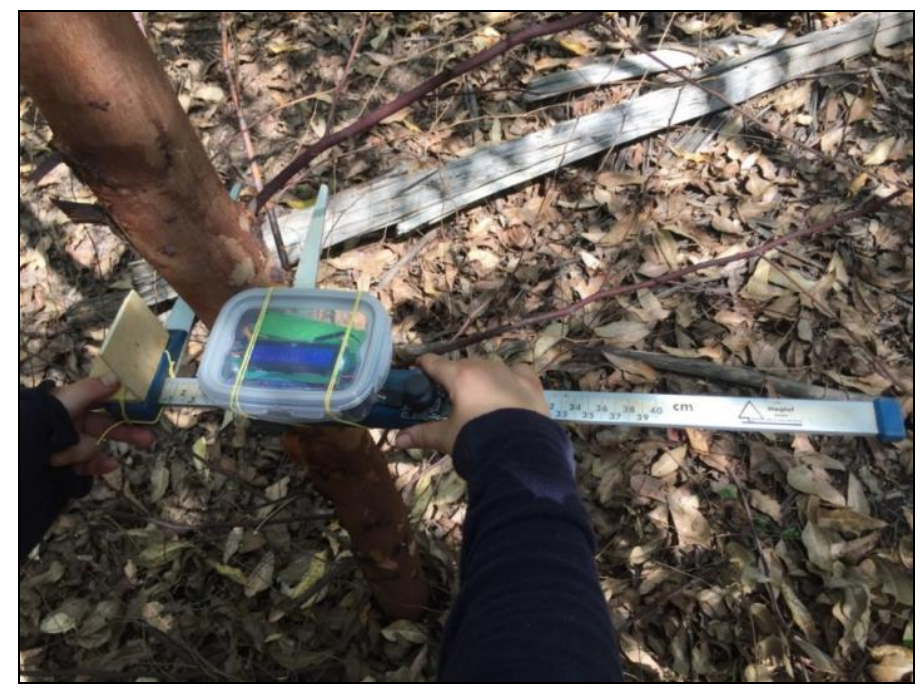

Fonte: Autores.

\section{Resultados e Discussão}

Ambas as medidas de diâmetro, frontal (r: 0,92) e lateral (r: 0,91) (Figura 7), apresentaram correlação positiva, indicando que os valores de DAP obtidos utilizando o protótipo construído são similares aos valores coletados pelo método convencional, utilizando suta mecânica.

Figura 7. Correlação entre as medidas obtidas através da suta convencional e o protótipo construído para diâmetro frontal e diâmetro lateral.

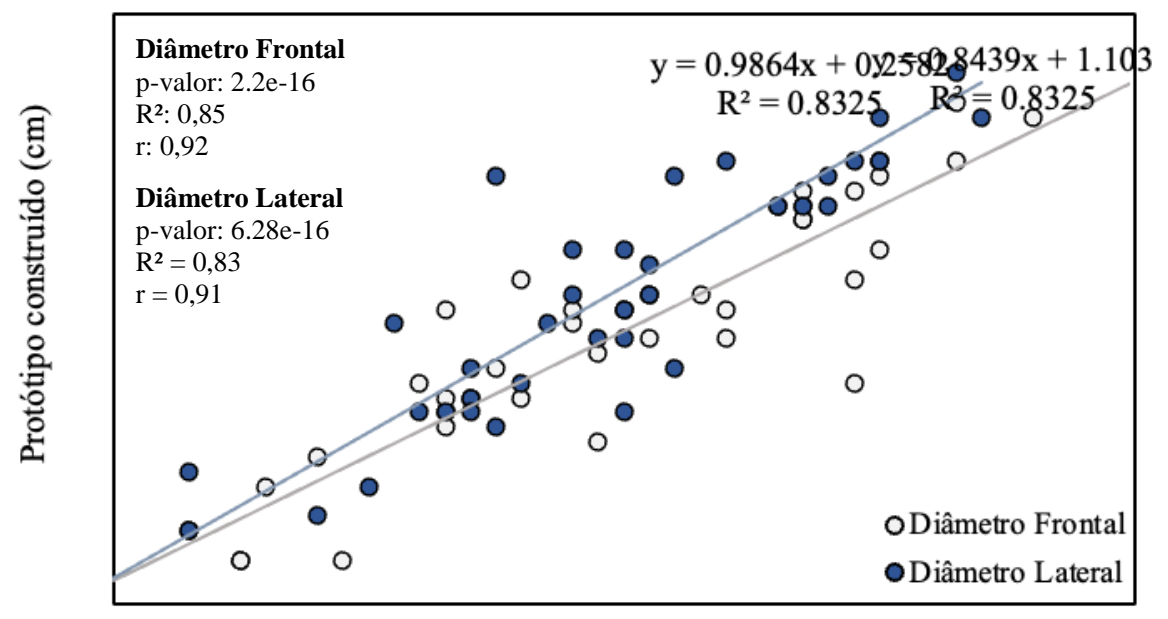

Suta convencional $(\mathrm{cm})$

Fonte: Autores.

A estrutura na qual os sensores foram instalados é um fator que dificulta o manuseio do instrumento (suta + protótipo) em campo, especialmente pelo fato de serem realizadas medidas de DAP frontal e DAP lateral para cada indivíduo presente na 
parcela. Além disso, o movimento do equipamento entre as medidas afetou a estabilidade dos dados no momento de sua obtenção.

Os principais benefícios da utilização do protótipo em campo foram: a possibilidade de acopla-lo em qualquer suta mecânica, a facilidade na aquisição dos dados e no seu armazenamento, diminuindo assim o uso de instrumentos para anotação, o tempo para coleta de dados e a necessidade de mão de obra. Além do custo para a confecção $R \$ 161,38$. O valor de confecção varia conforme o local de compra dos sensores e microcontrolador. As principais dificuldades observadas estão relacionadas à instabilidade do sensor durante a medição, que pode ser justificada pela falta de estabilidade da estrutura na suta e até mesmo pela própria estrutura.

Melhorias relacionadas a estrutura do protótipo são essenciais para a melhor estabilidade dos sensores e do posicionamento na suta mecânica. Estudos futuros serão realizados utilizando estruturas 3D e diferentes sensores a fim de reduzir de tornar a coleta dos dados mais precisa.

\section{Conclusão}

O protótipo desenvolvido utilizando sensores de baixo custo demonstrou eficiência na coleta de dados. O projeto se mostra viável para o uso dentro do setor florestal, principalmente em virtude o baixo custo para confecção e na facilidade de aquisição e armazenamento dos dados. No entanto, o protótipo apresentado necessita de maiores ajustes, principalmente em sua estrutura, a fim de facilitar o manuseio. Sugerimos a impressão em 3D para a estrutura em que os sensores forem alocados, a fim de reduzir dimensões e facilitar a trabalhabilidade.

\section{Agradecimentos}

À Josiana Jussara Nazaré Basílio, pelas contribuições feitas para a confecção deste projeto.

\section{Referências}

Araujo, H.J.B. (2006). Inventário florestal a 100\% em pequenas áreas sob manejo florestal madeireiro. Acta Amazonica. $36(4)$ p. 447 - 464.

Arduino. (2021). Documentação de Referência da Linguagem Arduino. https://www.arduino.cc/reference/pt/.

Arduino. (2020). Arduino Pro. https://www.arduino.cc/en/pmwiki.php?n=Main/ArduinoBoardProMini.

Campos, A., (2015) Arduino Pro Mini: conhecendo o modelo econômico. https://br-arduino.org/2015/02/arduino-pro-mini-conhecendo-o-modeloeconomico.html.

Corte, A. P. D., Sanquetta, C. R., Oliveira, K. A., Behling, A., \& Coutinho, V. M. (2016). Desempenho de diferentes equipamentos para mensuração de diâmetro a 1,30 m, altura individual total, e volume do fuste em Cryptomeria japonica (Thunb. ex L. f.) D. Don. Enciclopédia Biosfera. $13(23)$, p. 432.

Couto, H. T. Z. do, Batista, J. L. F., \& Rodriguez, L. C. E. (1989). Mensuração e gerenciamento de pequenas florestas. Piracicaba: Departamento de Ciências Florestais. ESALQ/USP. http://www.ipef.br/publicacoes/docflorestais/cap5.pdf

Cunha, S. U., Machado, S. A., \& Figueiredo Filho, A. (2002). Avaliação de erros não amostrais das variáveis locação (xi, yi) e diâmetro (cm) em inventários comerciais a $100 \%$ na floresta nacional do tapajós, Pará. Floresta e Ambiente. 9 (1), p.26-37.

Freitas, A. G., \& Wichert, M.C.P. (1998). Comparação entre instrumentos tradicionais de medição de diâmetro e altura com o Criterion 400. Circular Técnica IPEF. 188, p.1-7.

Gonçalves, D. A., Eldik, T. V., \& Pokorny, B. (2009). O uso de dendrômetros a laser em florestas tropicais: aplicações para o manejo florestal na Amazônia. Floresta. 39 (1), p.175-187.

Multilógica Shop. (2016). Arduino: Guia Iniciante. https://multilogica-shop.com/download_guia_arduino.

Murta, G. (2018). Guia completo do Display LCD - Arduino. https://blog.eletrogate.com/guia-completo-do-display-lcd-arduino/.

Nascimento, E. C., Silva, V. L,. \& Kashiwagi, M. (2019). Detecção de objetos em ambiente controlado por meio de mapeamento, utilizando uma plataforma robótica arduino. Anais da Mostra Nacional de Robótica - MNR 2019. http://sistemaolimpo.org/midias/uploads/c7ee98fc7511b32561a26dc4f367b919.pdf.

Oliveira, E. (2020). Como usar com Arduino - Módulo Joystick KY-023. https://blogmasterwalkershop.com.br/arduino/como-usar-com-arduino-modulojoystick-ky-023/. 
Research, Society and Development, v. 10, n. 17, e70101724197, 2021

(CC BY 4.0) | ISSN 2525-3409 | DOI: http://dx.doi.org/10.33448/rsd-v10i17.24197

Pereira, A.S., Shitsuka, D. M., Parreira, F. J., \& Shitsuka, R. (2018). Metodologia da pesquisa científica. Santa Maria/RS. Ed. UAB/NTE/UFSM.

Ribeiro, A.P., Rezende, B.N., Menegali, I., \& Pereira, S. (2016). Avaliação de temperatura e umidade utilizando o sensor DHT ${ }^{22}$ controlado por Arduino. Congresso Técnico Científico da Engenharia e da Agronomia (CONTECC). https://www.confea.org.br/sites/default/files/uploadsimce/contecc2016/agronomia/avalia\%C3\%A7\%C3\%A3o\%20de\%20temperatura\%20e\%20umidade\%20utilizando\%20o\%20sensor\%20dht22\%20controlado\% 20por\%20arduino.pdf

Sanquetta, C., Watzlawick, L., Dalla Côrte, A., Fernandes, L., \& Siqueira, J. (2009). Inventários Florestais: Planejamento e Execução. Curitiba: MultiGraphic Gráfica e Editora.

Silva, J. L. S., Melo, M. C., Camilo, R. S., Galindo, A., \& Viana, E. C. (2014). Plataforma Arduíno integrado ao PLX-DAQ: Análise e aprimoramento de sensores com ênfase no LM35. XIV Escola Regional de Computação Bahia, Alagoas e Sergipe (ERBASE).

Souza, F. (2013). Introdução ao Arduíno - Primeiros passos na plataforma. https://www.embarcados.com.br/arduino-primeiros-passos/.

Straub, M. G. (2018). Projeto arduino sd card: leitura e escrita de dados no cartão micro sd. https://www.usinainfo.com.br/blog/projeto-arduino-sd-cardleitura-e-escrita-de-dados-no-cartao-micro-sd/

Vidal, V. (2017). Gravação de cartão micro SD com Arduino - Faça seu Datalogger!. https://blog.eletrogate.com/gravacao-de-cartao-micro-sd-com-arduino/.

Waveshare. (2018). VL53L0X Distance Sensor User Manual. https://www.waveshare.com/w/upload/0/01/VL53L0X-Distance-Sensor-User-Manual-en.pdf. 